\title{
KINETIC EFFECTS IN THE SELF-ASSEMBLY OF PURE AND MIXED TETRADECYL AND OCTADECYLAMINE MOLECULES ON MICA
}

\author{
J.J. Benítez ${ }^{(1)}$ and M. Salmeron ${ }^{(2)}$.
}

${ }^{(1)}$ Instituto de Ciencia de Materiales de Sevilla. Centro Mixto CSIC.-

Universidad de Sevilla. Avda. Américo Vespuccio 49. Isla de la Cartuja.

Sevilla 41092 (Spain).

Email: benitez@icmse.csic.es

${ }^{(2)}$ Materials Science Division, Lawrence Berkeley National Laboratory.

University of California. Berkeley, California 94720.

Email: mbsalmeron@Ibl.gov 


\begin{abstract}
The self-assembly of tetradecylamine (C14) and of mixtures of tetradecyl and octadecylamine $(\mathrm{C} 18)$ molecules from chloroform solutions on mica has been studied using atomic force microscopy (AFM). For pure components selfassembly proceeds more slowly for $\mathrm{C} 14$ than for $\mathrm{C} 18$. In both cases after equilibrium is reached islands of tilted molecules cover a similar fraction of the surface. Images of films formed by mixtures of molecules acquired before equilibrium is reached (short ripening time at room temperature) show only islands with the height corresponding to $\mathrm{C} 18$ with many pores. After a long ripening time, when equilibrium is reached, islands of segregated pure components are formed.
\end{abstract}




\section{Introduction}

Most research on self-assembled monolayers (SAMs) using atomic force microscopy (AFM) has been performed using linear alkyl molecules with thiols or silane functional groups. Such groups provide strong chemical binding to substrates such as gold, silicon oxide, mica, and glass ${ }^{1,2,3,4,5,6,7,8}$. Alkyl molecules with amine groups on the other hand bind more weakly to the substrate and are more mobile ${ }^{9}$. The binding of amine molecules to the substrate is due to electrostatic forces (from $-\mathrm{NH}_{2}$ dipoles or charged $-\mathrm{NH}_{3}{ }^{+}$ groups), which are weaker than the covalent interactions prevalent in thiols and silanes. The films can easily form multilayers with the molecules in alternating orientations $^{10}$. We have previously shown that water plays an important role in the formation of such films. Indeed we found that self-assembly takes place on patches of residual water that contribute to the bonding with the substrate probably via formation of $-\mathrm{NH}_{3}{ }^{+} \mathrm{OH}^{-}$species.

In this paper we report results obtained with mixtures of alkylamine molecules of different alkyl chain lengths, with particular attention to the formation of homogeneous mixtures or segregated domains. The films are good models to study correlations between structural and mechanical properties of organic layers in fundamental studies of friction and lubrication ${ }^{11}$. For example, it has been shown that changes in molecular configuration due to mechanical interactions can be associated with energy dissipation events causing friction ${ }^{6}$. In compact monolayers these include rigid tilts of the alkyl chains. Other molecular rearrangements such as production of gauche defects, (transformation of the trans-configuration of the C-C bond into the cisarrangement by a $120^{\circ}$ rotation around the C-C axis), are sterically hindered 
and thus difficult to produce, while they are easily produced in diluted films where the bending of the molecular chain is not impeded by narrowly spaced neighbouring molecules. One way to test the formation of such internal gauche defects is by using mixtures of long and short chains. If homogeneously distributed, the long chains will protrude above the shorter ones and would make it possible the production of gauche defects either spontaneously driven by temperature or by the mechanical pressure exerted by and AFM tip. Such an effect was recently demonstrated in mixtures of thiols on gold ${ }^{12}$. In this paper we present results of a study of mixtures of long and short alkyl amines, with 14 and 14 carbon chains. As we will show, in this case segregation into separate domains is the preferred equilibrium structure of such films. This segregation however is strongly dominated by kinetics at room temperature.

\section{Experimental}

Tetradecylamine (C14) and Octadecylamine (C18) (Fluka, >99\%) were used as received and dissolved in chloroform (Aldrich 99.8\%) to obtain solutions $15 \mathrm{mM}$ total concentration. Samples (approx. $3 \times 1 \mathrm{~cm}$ ) of muscovite mica $\left(\mathrm{KAl}_{2}\left(\mathrm{Si}_{3} \mathrm{AlO}{ }_{10}\right)(\mathrm{OH})_{2}\right.$ (Mica New York corp.) were cleaved on both sides at ambient conditions (typically $20^{\circ} \mathrm{C}$ and $50-55 \% \mathrm{RH}$ ) and quickly immersed in the solution for 30 seconds. The samples were subsequently removed and dried without rinsing under a $\mathrm{N}_{2}$ stream for several minutes. They were ripened inside a test tube until analyzed.

The atomic force microscope (AFM) head is home built and operates inside a box that provides sound isolation and humidity control. It is controlled by RHK Technology electronics and software. We used cantilevers 
(NanoProbes ${ }^{\mathrm{TM}}$, Digital Instruments) with $0.12 \mathrm{~N} / \mathrm{m}$ nominal force constant. The $z$ distance scale (normal to the surface) was calibrated using test silicon gratings (TGZ01, Silicon-MDT, Moscow) with step heights of $25.5 \mathrm{~nm}$, and also in situ by generating a $1 \mathrm{~nm}$ deep wear hole on the mica surface after scanning under a large load ${ }^{13}$. Since the maximum area scanned in our instrument is $5 \times 5 \mu \mathrm{m}^{2}$, to obtain a representative value of surface coverage, several points of the same sample have been analyzed and averaged.

\section{Results and discussion}

Tetradecylamine self-assembly

In contrast to the results obtained in our previous work with octadecylamine $^{9}$, which showed the ready formation of monolayer islands, no islands were found after immersion of mica in chloroform solutions of the shorter tetradecylamine molecules, even after overnight ripening. Instead the images showed a noisy and featureless surface, as in figure $1 \mathrm{~A}$. Approach and separation force curves obtained on such samples indicated that although not visible in the AFM images, the surface of mica has been modified by the immersion in the C14 solution. The pull-off force (curve 2) was considerably lower than on freshly cleaved mica (curve 1). We found also that the "noise" in the surface corresponds to a corrugation of 0.2 to $0.5 \mathrm{~nm}$ (line profile 2 ). These observations point to the presence of scattered tetradecylamine molecules lying flat on the surface of mica. Indeed, after the samples were allowed to ripen for longer periods of time (30 hours) islands were found, as shown in figure 1B, with a height of about $0.3 \mathrm{~nm}$. We attribute them to flat-lying tetradecylamine molecules. 
If ripening is allowed to continue for two days, islands with a height of 1 $\mathrm{nm}$ developed, as shown in figure 1C. As observed in the case of octadecylamine molecules investigated previously the molecular orientation is not vertical because the full length of the tetradecylamine molecules is $\sim 1.8 \mathrm{~nm}$. Simple geometry indicates that the $1 \mathrm{~nm}$ height corresponds to a $58^{\circ}$ tilt of the molecules from the surface normal. It was shown that linear alkyl molecules in SAM islands adopt discrete and well-defined tilt states $(\theta)$ given by the equation $^{6}$

$$
\tan \theta=\text { na } / d \quad n=0,1,2, \ldots \ldots
$$

where (a) is the distance between alternating $\mathrm{C}$ atoms in the alkyl molecule projected along the chain axis $(0.24 \mathrm{~nm}),(\mathrm{d})$ is the separation between packed alkyl molecules $(0.45 \mathrm{~nm})$ and $\mathrm{n}$ an integer, with 0 corresponding to fully vertical molecules. The $58^{\circ}$ tilt found for our molecules corresponds to $n=3$.

The tetradecylamine SAM islands were found to be more "fragile" than those formed by the longer octadecylamine. Repetitive scanning over the same area tends to displace the islands towards the edges of the scanned region. This can already be seen in figure $1 \mathrm{C}$, where the central region has been depleted of islands due to previous scans. This can be explained by the weaker van der Waals attraction between molecules as compared to the longer octadecylamine. This attraction is a key factor determining the growth and the stability of the islands. The island formed by the shorter tetradecylamine molecules should also be more compliant to the forces exerted by the tip, both in terms of being easier to tilt and to disrupt. 
Assembly of tetradecyl and octadecylamine mixtures

Figure 2 shows the images of SAM islands obtained from different mixtures of $\mathrm{C} 14$ and $\mathrm{C} 18$ after ripening for 14 hours. It is interesting to notice that large but highly defective (porous) islands are obtained in the $2: 8$ to $5: 5$ C14:C18 mixture range. The height of the islands is always the same $(1.6 \mathrm{~nm})$ for all mixture ratios and corresponds to that observed for islands obtained from pure octadecylamine solutions. The islands formed from octadecylamine-rich mixtures $(\mathrm{C} 14: \mathrm{C} 18=0: 10-2: 8)$ coexist with smaller and more compact ones, but disappear as the amount of $\mathrm{C} 14$ in the mixture is increased as shown in figure 2. The main observation is that as the fraction of $\mathrm{C} 14$ increases, the number of visible islands decreases to the point that no islands are observed when the $\mathrm{C} 14: \mathrm{C} 18$ ratio is above $8: 2$, for such preparation and ripening conditions. If the fractional area of mica covered by islands is plotted versus C14 molar ratio (figure 3), a linear decrease is observed.

We explain these results by considering the higher cohesive energy of the longer alkyl chain molecules. The kinetics of island formation depends on two important parameters: diffusion of isolated molecules over the mica surface and binding of these molecules into larger islands of upright (albeit tilted) molecules. Long chains have higher cohesive energy and lower diffusivity, while in the shorter chains cohesion is lower and diffusivity higher. This favours C18 to form SAM islands. The larger van der Waals cohesion energy of the C18 molecules is due to its additional 4 methylene groups over the $\mathrm{C} 14$. This contribution was calculated to be close to $78 \mathrm{meV}$ per methylene unit ${ }^{14}$, giving a total of $312 \mathrm{meV}$ more energy per molecule. 
The $\mathrm{C} 18$ islands formed from mixtures are smaller or have more pores (internal holes) than when prepared from pure $\mathrm{C} 18$ solutions because of intercalated C14 molecules, which decrease the number of direct $\mathrm{C} 18-\mathrm{C} 18$ contacts. When the $\mathrm{C} 14: \mathrm{C} 18$ ratio is too high no islands are formed because the collision rate between two or more $\mathrm{C} 18$ molecules has decreased to the point that the growth of nuclei is too low.

These observations however refer to conditions far from equilibrium, where diffusion and aggregation phenomena prevail. As we can see, these kinetic phenomena have time constants measured in hours and days in the present case. We already saw in figure 1 that after a ripening period of more than one day C14 SAM islands are formed from pure tetradecylamine solutions. Figure 3 shows that after a few days ripening the fractional area covered by C14 molecules is similar to that with pure C18. The independence of surface coverage with alkyl chain length, after equilibrium is reached, is in concordance with the crucial role of residual water in the bonding of alkylamines to mica. It is the patches of surface covered with water that act as nucleation sites for the standing up alkylamine molecules.

Similar fractional coverage is reached after sufficient ripening time (few days) for all mixtures in figure 3. Interestingly the islands obtained such mixtures segregate into regions of pure $\mathrm{C} 14$ and pure $\mathrm{C} 18$, as shown in figure 4. The $\mathrm{C} 14$ phase regions are always found next to $\mathrm{C} 18$ regions and no isolated islands of pure $\mathrm{C} 14$ were observed, except with the pure component. This result indicates that the $\mathrm{C} 14$ molecules segregate from previously mixed islands, forming small pockets inside or at the periphery of the islands. The 
edges of the more stable C18 islands can act also as anchoring points for the more mobile C14 molecules allowing them to nucleate and grow there.

Another interesting observation is that although separately the alkylamine molecules can adopt a variety of discrete tilt angles, in the segregated islands of mixtures, both long and short molecules exhibit the same tilting angle, characterized by the integer $n=2\left(\theta=47^{\circ}\right)$. This is easily deduced by comparing the island heights $(1.55$ and $1.30 \mathrm{~nm})$ with the predictions of formula [1], 1.66 and $1.29 \mathrm{~nm}$. The alkyl chains at the boundary separating the tetradecyl and octadecyl amines are indeed parallel to each other, thus favouring maximum contact area and sharing the same tilt angle.

Separately, both $\mathrm{C} 14$ and $\mathrm{C} 18$ can also tilt to the next state $(n=3)$ when allowed to ripen for a much longer period of time, although the longer alkylamines take longer time.

In comparison with the results of mixed C12 and C16 alkylthiols on $A u(111)$, which do not segregate into separate domains even after ripening times of 48 hours, the segregation exhibited by the mixed C14-C18 alkylamines on mica points to differences in the binding to the substrate as the major cause for the different behaviour. The stronger covalent S-Au bonding results in a lower molecular mobility than in the $\mathrm{NH}_{3}{ }^{+} \mathrm{OH}^{-}$mica case studied here.

\section{Conclusions}

The kinetics of self-assembly of linear alkylamines is a strong function of the alkyl chain length. Compared with octadecylamine, tetradecylamine molecules with only 4 methylene units less, need considerably more ripening 
time to form islands. Even when formed the tetradecylamine islands can be much more easily displaced with the AFM tip in contact mode.

When mixed, both alkylamine molecules are adsorbed on mica although tetradecylamine does not assemble as fast as octadecylamine. Thus, after short periods of ripening only defective islands with height corresponding to C18 are observed with AFM. In the mixed layers and after sufficient ripening time to reach equilibrium islands with segregated areas of C18 and C14 form with all molecules adopting the same tilting angle. The fractional area of mica covered by alklyamines under equilibrium conditions is roughly independent of the mixture ratio and is dictated mostly by the fractional area covered with water.

\section{Acknowledgements}

This work was supported by the Director, Office of Energy Research, Office of Basic Energy Sciences, Material Science Division, of the U.S. Department of Energy under Contract No. DE-AC03-76SF00098. JJB acknowledges the financial support from the Spanish Ministerio de Educación, Cultura y Deporte under the "Movilidad del Profesorado Universitario e Investigadores" program. 


\section{Figure Captions}

Figure 1. (A) $2.5 \times 2.5 \mu \mathrm{m}$ AFM image of mica acquired after 14 hours immersion in a tetradecylamine chloroform solution. No molecular structures are observed. The pull-off force curves on the right graph correspond to freshly cleaved mica (1) and to mica exposed to the solution (2). The images after immersion (B) show a noisy topography with bumps of a few angstroms in height (see profiles on the right). After ripening for 30 hours (B) and 48 hours $(C, 5 \times 5 \mu \mathrm{m})$ islands are formed. Line profiles on the right hand side indicate that islands of flat lying molecules form first (1), which later tilt up into more vertical configurations with an inclination of $58^{\circ}$ from the normal.

Figure 2. $2.5 \times 2.5 \mu \mathrm{m}$ AFM images showing islands obtained from tetradecyl: octadecylamine mixtures after 14 hours ripening at room temperature. The molecular ratio of the mixture used in each case is indicated above each image.

Figure 3. Fractional area of mica covered by islands visible in the AFM images versus tetradecyl:octadecylamine molar ratio in the chloroform solution after a ripening time of 14 hours. No islands are observed above a C14:C18 $=0.8$ molar ratio for ripening times below 14 hours. However, when allowed to ripen for four days a similar $30 \%$ coverage is achieved, even for pure $\mathrm{C} 14$.

Figure 4. AFM images showing islands formed after immersion into a mixed chloroform solution of tetradecyl and octadecylamine molecules. Segregation into pure $\mathrm{C} 14$ and $\mathrm{C} 18$ regions is observed after four days ripening. The two 
examples shown here correspond to $\mathrm{C} 14: \mathrm{C} 18=1: 9$ and 5:5 mixtures. The heights measured by the line profiles indicate that the segregated regions, despite the length difference, consist of molecules with the same tilt angle of $47^{\circ}$ with respect to the surface normal direction.

\section{References}

${ }^{1}$ A. Ulman. "Thin Films: Self Assembled Monolayers of Thiols". Academic Press, New York (1998).

${ }^{2}$ F. Schreiber. Prog. Surf. Sci., 65, 151 (2001).

${ }^{3}$ D. Allara, A.N. Parikh and F. Rondelez. Langmuir, 11, 2357 (1995).

${ }^{4}$ A. Ulman. Chem. Rev., 96, 1533 (1996).

${ }^{5}$ C. Kessel and S. Granik. Langmuir, 7, 532 (1991).

${ }^{6}$ E. Barrena, S. Kopta, D.F. Ogletree, D.H. Charych and M. Salmeron. Phys. Rev. Lett., 82, 2880 (1999).

${ }^{7}$ X.D. Xiao, J. Hu, D.H. Charych and M. Salmeron. Langmuir, 12, 235 (1996).

${ }^{8}$ A. Lio, D.H. Charych and M. Salmeron. J. Phys. Chem. B, 101, 3800 (1997).

${ }^{9}$ J.J. Benítez, S. Kopta, D.F. Ogletree and M. Salmeron. Langmuir, 18, 6096 (2002).

10 J.J. Benítez, S. Kopta, I. Díez-Pérez, F. Sanz, D.F. Ogletree and M.

Salmeron. Langmuir, 19, 762 (2003).

${ }_{11}^{11}$ R.W. Carpick and M. Salmeron. Chem. Rev., 97, 1163 (1997).

12 E. Barrena, C. Ocal and M. Salmeron, Surf. Sci., 482-485, 1216 (2001).

${ }^{13}$ S. Kopta and M. Salmeron. J. Chem. Phys., 13 (18), 8249 (2000).

${ }^{14}$ M. Salmeron, Trib. Lett., 10, 69 (2001). 

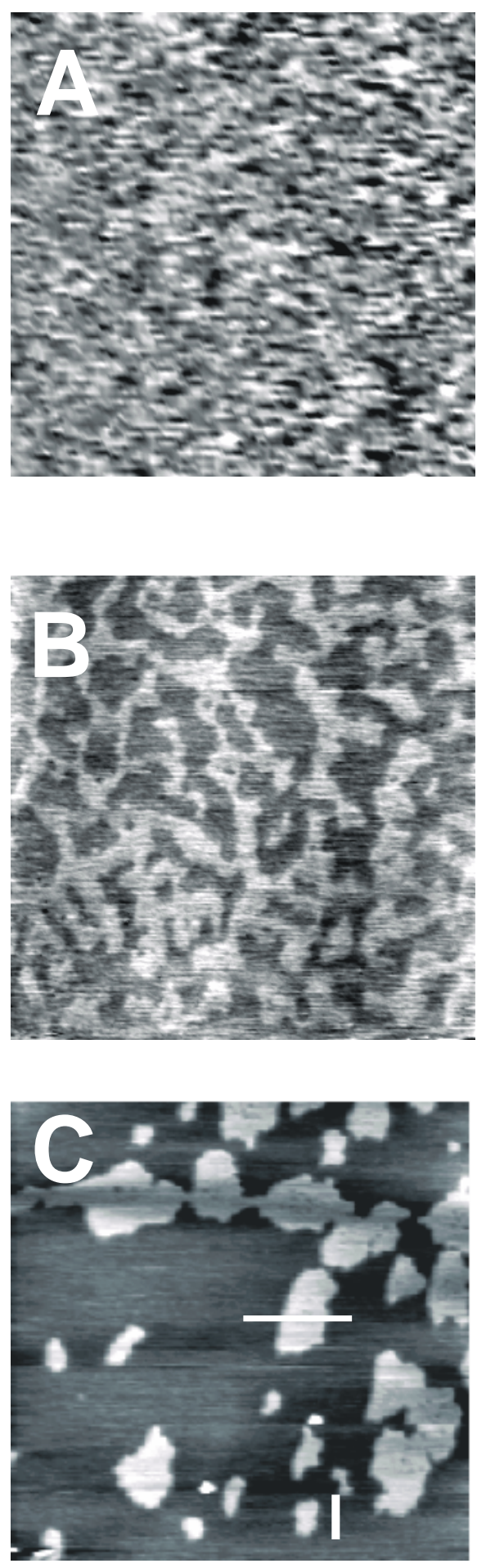
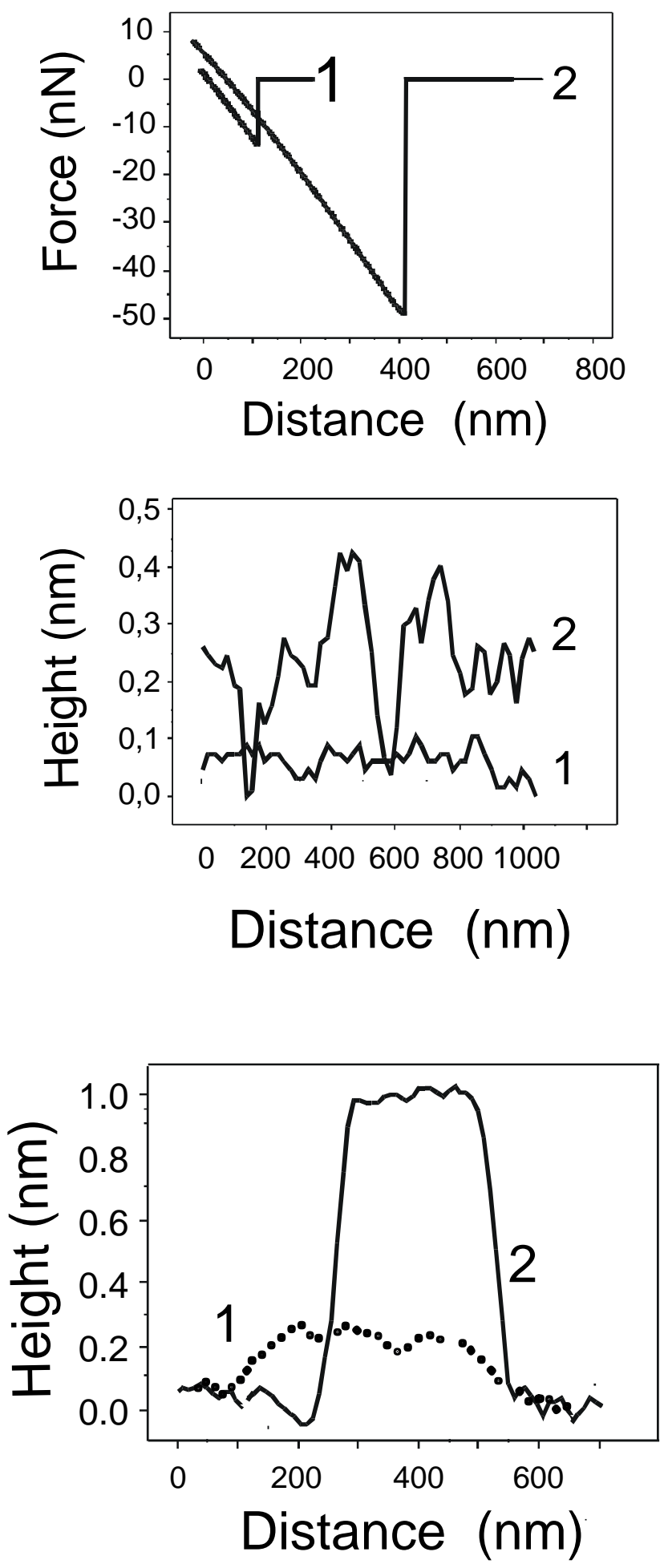


\section{$0: 10$ \\ 1:9}
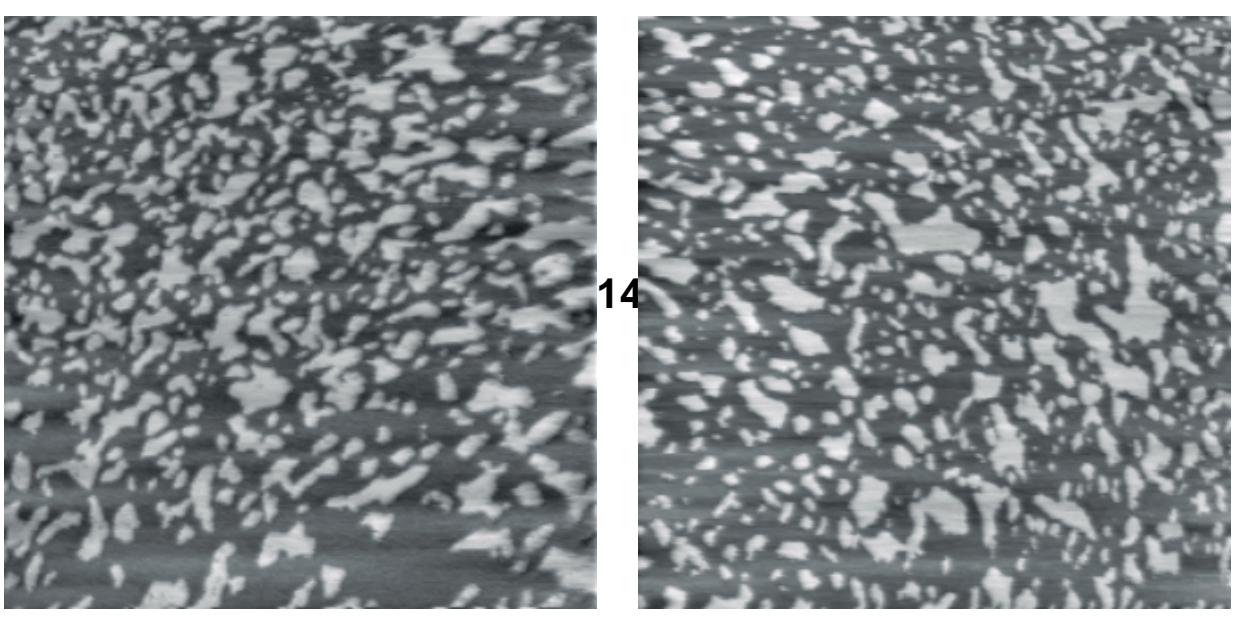

\section{$2: 8$}

\section{$3: 7$}
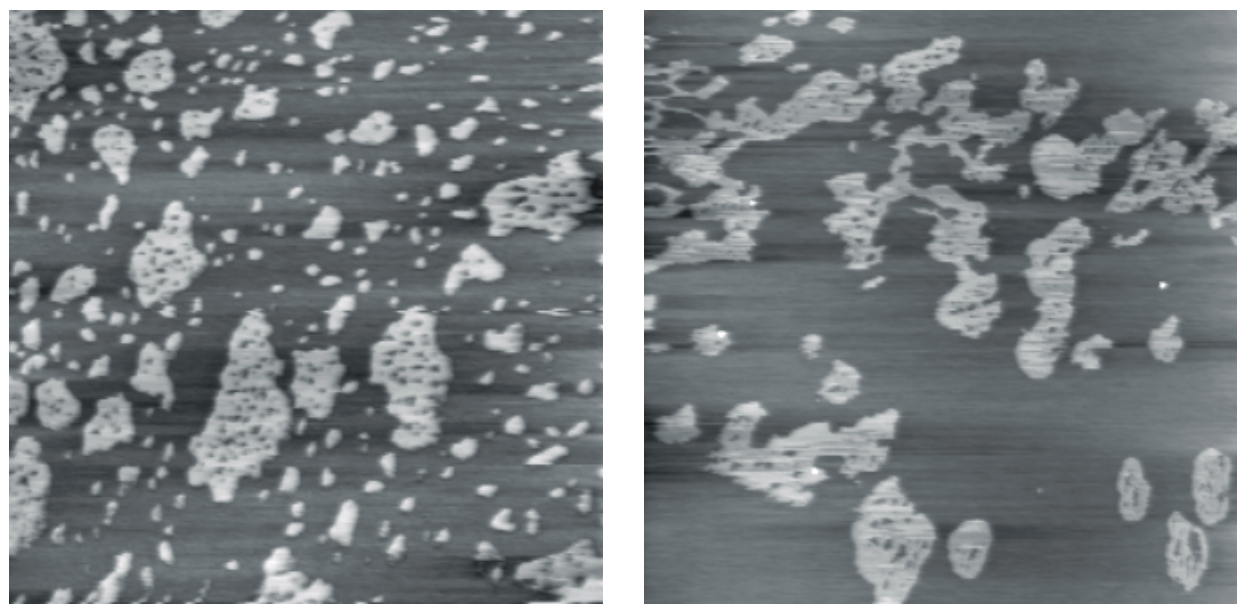

\section{5:5}

$7: 3$
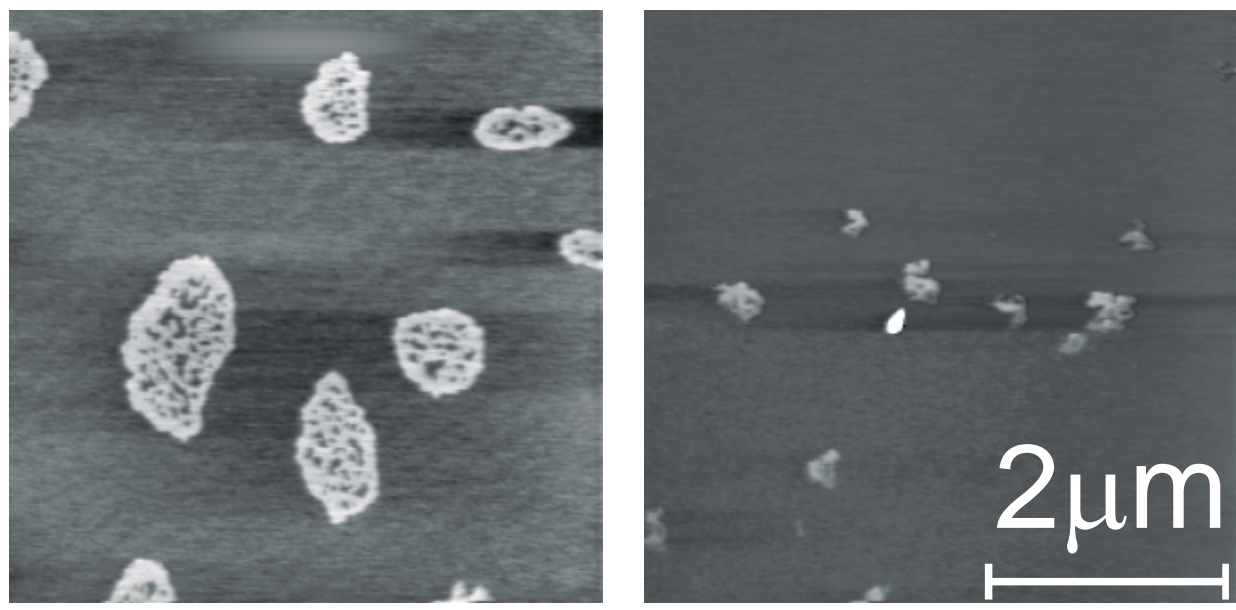


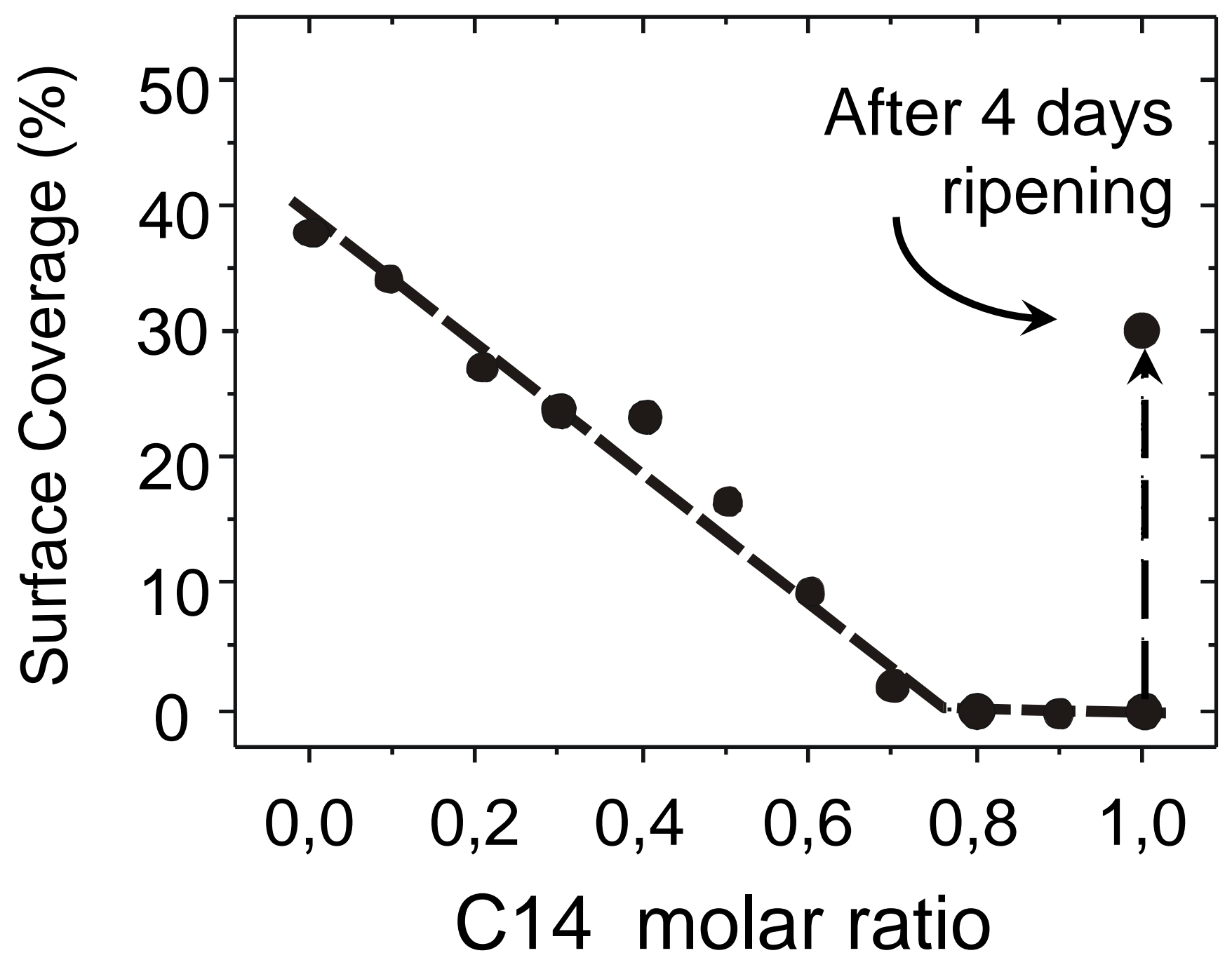




\section{C14:C18 = 1:9}

\section{$\mathrm{C} 14: \mathrm{C} 18=5: 5$}
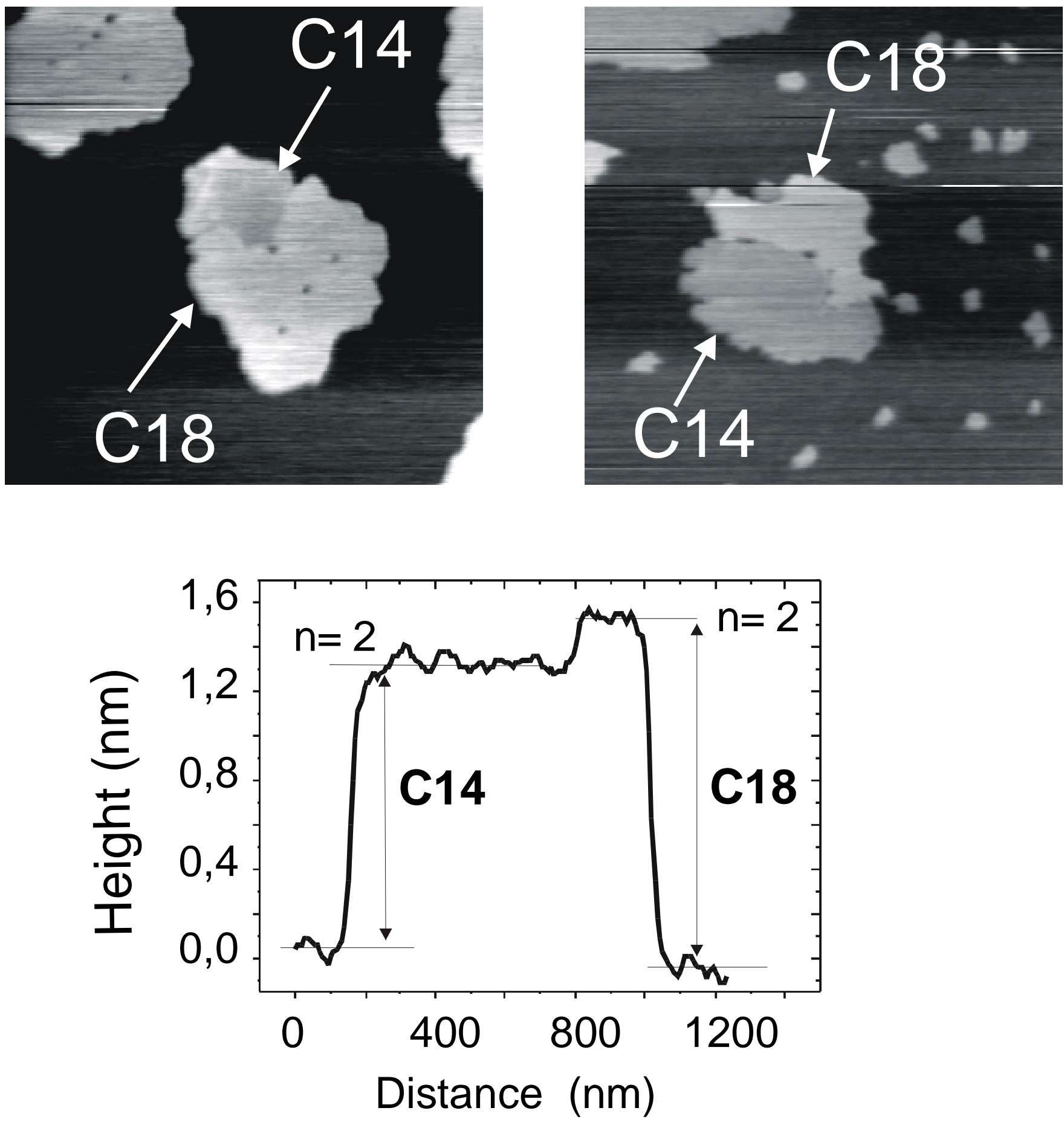